INVESTIGACIONES

\title{
LA EDUCACION CIENTIFICA COMO APOYO A LA MOVILIDAD SOCIAL: DESAFIOS EN TORNO AL ROL DEL PROFESOR SECUNDARIO EN LA IMPLEMENTACION DE LA INDAGACION CIENTIFICA COMO ENFOQUE PEDAGOGICO*
}

\author{
Scientific education as a support of social mobility
}

\section{Corina González Weil, María Teresa Martínez Larraín, Carolina Martínez Galaz, Karen Cuevas Solís, Liber Muñoz Concha}

Pontificia Universidad Católica de Valparaíso, Avda. Brasil 2950, Valparaíso.

corina.gonzalez@ucv.cl

\section{Resumen}

La educación es uno de los principales factores que promueven la movilidad social, siendo la educación secundaria clave, tanto para el desarrollo de procesos cognitivos superiores como para la definición del destino de los individuos, una vez que egresan del sistema escolar. La educación científica, mediante la generación de competencia científica, puede colaborar fuertemente en este desarrollo. A nivel tanto internacional como nacional se promueve el uso de la indagación científica como un enfoque pedagógico efectivo en el desarrollo de estas competencias, especialmente bajo contextos de alta vulnerabilidad. El presente trabajo expone algunos rasgos claves que debiera tener el profesor para implementar este enfoque. Finalmente se reflexiona acerca del rol de las universidades formadoras de profesores en el logro de este desafío.

Palabras clave: competencia científica, Enseñanza de las ciencias, indagación científica, educación secundaria, equidad.

\begin{abstract}
Education is one of the main factors that promote social mobility. Secondary education is important for the development of upper cognitive processes as for the destiny of the individuals, once they graduate of the school system. Scientific education, by means of the generation of scientific competence, can collaborate strongly in this development. At international and national level, the use of scientific inquiry is promoted as an effective pedagogical focus in the development of these competences, especially, at contexts of high vulnerability. The present work exposes some key features that teachers should have to implement this pedagogical focus. Finally, the role of science secondary teacher education is discussed.
\end{abstract}

Key words: scientific competence, science education, scientific inquiry, secondary education, equity.

\footnotetext{
* Este trabajo se desarrolla en el marco de los proyectos de investigación DI 122.799 /2007 y DI 037.107/2008, financiados por la Dirección de Investigación e Innovación de la Pontificia Universidad Católica de Valparaíso.
} 


\section{INTRODUCCION}

Existe suficiente evidencia para afirmar que la generación de capital humano a través de la educación constituye uno de los principales factores que promueven la movilidad social. Adicionalmente, la educación secundaria, particularmente en América Latina, parece ser una etapa crucial para los procesos de desarrollo social y para el destino personal de los individuos (Tedesco y López 2002). No obstante su importancia, este tramo educativo concentra el mayor desfase entre lo que la sociedad espera del proceso educacional y lo que en realidad se ofrece. Así, aunque a menudo los jóvenes y sus familias vean en la educación secundaria la vía de acceso a la promoción social y económica, este tramo suele ser inequitativo y preparar insuficientemente a los estudiantes tanto para seguir estudios superiores como para la entrada al mercado laboral (Macedo y Katzkowicz 2002). Bajo este contexto, la imagen que la sociedad y a menudo los propios profesores tienen de estos alumnos cambia desde una mirada asistencialista de "niños a los que hay que proteger y comprender" en las escuelas, a una mirada de exclusión, considerándolos "jóvenes marginales" causantes de la mayoría de los "problemas sociales" (Haberman 2006). De este modo, no es extraño que en muchos profesores de liceos en contextos de alta vulnerabilidad se observe una tendencia preferente por "transmitir la materia", más allá del logro de aprendizajes profundos, bajo la premisa de que "aquel alumno que esté interesado, aprenderá, el que no, pues no", atribuyendo los malos resultados de aprendizaje a la situación de pobreza, a las condiciones del hogar, la familia o a la mala formación recibida durante la educación básica, y aceptando como natural el hecho de que los alumnos repliquen el destino de sus padres (Haberman 2006).

En el ámbito de la educación científica en particular, los profesores comúnmente le dan más importancia a los contenidos que al desarrollo de habilidades y actitudes, dominando las metodologías tradicionales, basadas fuertemente en la lectura de libros de texto, y la instrucción directa del profesor, por sobre las actividades de indagación científica y el trabajo de campo (Vergara 2006, Krugly-Smolska 1990). No obstante lo anterior, existen docentes que con niños en contextos de similar vulnerabilidad realizan otro tipo de actividades y obtienen resultados de aprendizaje exitosos. ¿Qué hace la diferencia? Según Haberman (2006) y Zahur et al. (2002), este tipo de profesores, además de creer en sus alumnos, creen en la educación científica como promotora de un cambio social. Según su visión, la educación científica ayudaría a los alumnos, en aquellas comunidades más pobres, a ganar voz y espacio en la sociedad, además de elevar la calidad de vida, tanto de los propios alumnos como de su comunidad. En los siguientes párrafos se describen algunos aspectos clave del profesor, que hacen posible pensar la enseñanza de las ciencias como apoyo a la movilidad social, en el contexto de la educación secundaria.

\section{LA IMPORTANCIA DE ENSEÑAR CIENCIAS}

La ciencia ocupa hoy un rol protagónico en la sociedad. El conocimiento científico ha trascendido prácticamente a todos los aspectos de lo cotidiano, y se vuelve indispensable no sólo para la comprensión del medio en que estamos inmersos, sino también para participar de manera fundamentada en una sociedad democrática. Es así que en la Conferencia Mundial sobre la Ciencia para el siglo XXI (año 1999), auspiciada por la 
UNESCO, se considera que "el acceso al saber científico con fines pacíficos desde una edad muy temprana forma parte del derecho a la educación que tienen todos los hombres y mujeres, y que la enseñanza de la ciencia es fundamental para la plena realización del ser humano, para crear una capacidad científica endógena y para contar con ciudadanos activos e informados". Y proclama: "La enseñanza científica, en sentido amplio, sin discriminación y que abarque todos los niveles y modalidades, es un requisito previo esencial de la democracia y el desarrollo sostenible" (Declaración de Budapest, UNESCO-ICSU, 1999). Del reconocimiento que hace la comunidad internacional acerca de la importancia de la ciencia en la vida cotidiana de las personas, es que surge el término Alfabetización Científica, definida por el Grupo de Expertos del área de Ciencias del PISA (Programme for International Student Assessment, de la OECD") como "la capacidad de usar el conocimiento científico para identificar preguntas y para sacar conclusiones basadas en las pruebas, con el fin de entender y ayudar a tomar decisiones sobre el mundo natural y los cambios realizados en él a través de la actividad humana" (Harlen 2002: 210). La importancia de la alfabetización científica de la población es reconocida internacionalmente. En palabras de Macedo y Katzkowicz (2005: 1), "hoy en día no podemos separar la alfabetización científica y tecnológica del concepto de alfabetización general ya que la cultura científica contribuye a la formación ciudadana, al desarrollo social y económico de las sociedades y por lo tanto a la inclusión y la equidad social".

Esta concepción, internacionalmente consensuada, supone un cambio en el objetivo de la educación científica. Si antes el énfasis estaba puesto en el generar nuevos científicos, ahora el acento se coloca en lograr en todas las personas un grado suficiente de conocimientos, habilidades y actitudes científicas, entendiendo que esto es relevante para la vida de cualquier ciudadano. En los países en vías de desarrollo la educación científica debiera suponer especialmente un mejoramiento de la calidad de vida de todos los ciudadanos, por ejemplo, al enfatizar aspectos como la salud y la nutrición, el cuidado del medio ambiente, el desarrollo de competencias para el trabajo, etc. Así, en las comunidades más vulnerables, como es el caso de muchos de nuestros establecimientos municipales y subvencionados, una educación científica de calidad, que traiga conocimientos científicos, tecnológicos y matemáticos, puede constituir un factor crítico para el logro de justicia social (Zahur et al. 2002).

En el contexto de un individuo en particular, y siguiendo el marco teórico de PISA 2006, el estar alfabetizado científicamente supone haber desarrollado Competencia Científica, lo que hace referencia a: (a) el conocimiento científico y el uso que se hace de ese conocimiento para identificar preguntas, adquirir nuevos conocimientos, explicar fenómenos científicos y extraer conclusiones basadas en pruebas sobre temas relacionados con las ciencias, (b) la comprensión de los rasgos característicos de la ciencia, entendida como una forma del conocimiento y la investigación humanos, (c) la conciencia de las formas en que la ciencia y la tecnología moldean nuestro entorno material, intelectual y cultural, (d) la disposición a implicarse en asuntos relacionados con la ciencia y a comprometerse con las ideas de la ciencia como un ciudadano reflexivo (OECD 2006).

1 Organización Económica de Cooperación y Desarrollo. 


\section{LOS NIVELES DE ALFABETIZACION CIENTIFICA EN CHILE}

A pesar de su importancia, los resultados de la educación científica en Chile merecen especial atención, particularmente en cuanto a las condiciones de equidad en el aprendizaje. El Programa PISA de la OECD constituye actualmente uno de los instrumentos más relevantes en la medición del grado de alfabetización científica/competencia científica de los jóvenes. En PISA se evalúa lo que los países participantes han acordado como los resultados deseables de la educación científica, estén o no reflejados en los currículos de cada país. Esta prueba es aplicada a alumnos de 15 años ( $2^{\circ}$ medio en Chile) y ha sido realizada en 43 países el 2000, 41 países en el 2003 y en 57 países el 2006, participando en cada caso entre 4.500 y 10.000 alumnos por cada país ${ }^{2}$. En Chile, los resultados de PISA $2000^{3}$ indicaron que el desempeño promedio de los alumnos de $2^{\circ}$ medio evaluados está asociado al nivel de tareas más básicas de ciencias en el marco de PISA 2000. Según éste, los estudiantes usualmente son capaces de recordar conocimientos científicos simples, como, por ejemplo, nombres, hechos o terminología, además pueden usar conocimiento científico común para elaborar o evaluar conclusiones, pero no logran usar conceptos científicos ni crear modelos conceptuales para hacer predicciones o dar explicaciones. Tampoco pueden identificar detalles de los aspectos relacionados con una investigación científica, ni seleccionar información de cadenas de razonamiento que sean útiles para obtener y evaluar conclusiones (MINEDUC 2003). Si bien los resultados de Chile en PISA 2006 fueron mejores, mostrando un desempeño superior al de estudiantes de los países latinoamericanos participantes del estudio, se debe reconocer que el desempeño de los estudiantes chilenos (438 puntos promedio) sigue siendo significativamente inferior que el promedio de estudiantes de la OECD (500 puntos) y de los de países comparados pertenecientes a ella (MINEDUC 2006). Más aún, existe una significativa brecha entre el desempeño promedio de los alumnos del quintil más bajo y los del quintil más alto consistente en 124 puntos, lo que refleja inequidad en cuanto a los resultados de aprendizaje, originados ciertamente por una cuestión de índice socioeconómica y cultural (MINEDUC 2006) que coloca en desventaja a aquellos estudiantes provenientes de contextos privados de oportunidades. Estos resultados coinciden con los de SIMCE 2007: el desempeño de los alumnos se relaciona claramente con su nivel socioeconómico. De esta manera, y en general, los alumnos que presentan los resultados más bajos son los más pobres (SIMCE 2008; Albertini et al. 2005).

Muchos países no quedaron satisfechos con el desempeño de sus estudiantes en PISA. En vista de ello, existen contribuciones de distintos autores en relación a las competencias que debe tener un profesor para poder revertir esta situación y así cumplir con los nuevos objetivos propuestos en la educación científica.

2 Hasta el año 2003 sólo se medían conocimientos y habilidades, pero no actitudes, por lo que no se puede hablar aún de medición de la "competencia científica". Recién el año 2006 se incorpora este último aspecto.

3 PISA se aplicó el año 2000 en 32 países. Después de iniciado el estudio, la OECD realizó un proyecto paralelo denominado PISA +, para permitir que otros países interesados participaran en 2001. Ese fue el caso de Chile. Dado que los objetivos, metodología y población evaluada en este segundo estudio replicaron exactamente al primero fue posible comparar los resultados entre los 43 países participantes entre 2000 y 2001. 


\section{EL ROL DEL PROFESOR EN EL LOGRO DE COMPETENCIA CIENTIFICA EN SUS ALUMNOS}

Según Hattie (2003), Román (2003) y Marcelo (2006), múltiples investigaciones dan cuenta del rol protagónico del profesor en los logros de aprendizaje de sus alumnos, lo cual lo sitúa como principal responsable de la alfabetización científica de los estudiantes. Esto parece ser especialmente relevante en el caso de los profesores de enseñanza media, si se considera, según Gil y Vilches (2001), que la educación secundaria constituye la etapa fundamental para plantear la alfabetización científica de los futuros ciudadanos y ciudadanas. Si bien es poco probable que haya desacuerdo respecto de los objetivos actuales de la educación científica, en la práctica el énfasis de la enseñanza está en los contenidos más que en el desarrollo de habilidades y actitudes (Krugly-Smolska 1990). A nivel nacional, la formación científica del alumno, particularmente en el liceo, se caracteriza por un aprendizaje memorístico de contenidos disgregados, con una comprensión de la ciencia descontextualizada y alejada de lo cotidiano (Albertini et al. 2005). Sin embargo, si se considera el carácter dinámico de la ciencia, nos encontramos con que los contenidos son también dinámicos, y cambian con el tiempo. De esta manera, parece ser que lo fundamental en la educación científica es "enseñar ciencia como una manera de conocer, con consecuencias prácticas" (Krugly-Smolska 1990: 478) más que enseñarla como un conjunto de conocimientos cerrado. A partir de lo anterior, y considerando el rol protagónico del profesor, parece imperativo el reformular los métodos de enseñanza de las ciencias.

La indagación científica como enfoque pedagógico. El término "indagación científica" ha sido utilizado ampliamente en la educación de las ciencias. Windschitl lo define como un proceso en el cual "se plantean preguntas acerca del mundo natural, se generan hipótesis, se diseña una investigación, y se colectan y analizan datos con el objeto de encontrar una solución al problema" (Windschitl 2003: 113). El concepto "Indagación científica" puede ser entendido como objetivos de aprendizaje, una metodología de enseñanza o un enfoque pedagógico, es decir, un conjunto de conocimientos y creencias que guían la enseñanza de las ciencias (Abell et al. 2006). Desde una perspectiva sociocultural, puede entenderse como "maneras de generar explicaciones, cargadas de teoría, validadas por una comunidad, apoyadas por evidencia y argumentos convincentes y mantenidas por la comunidad como conocimiento tentativo y abierto a futuros desarrollos" (Abell et al. 2006: 174). En el campo educativo, la indagación científica del modo en que se describe constituye un camino plausible mediante el cual el alumno puede construir su propio conocimiento, pensar acerca de lo que sabe, y acerca de cómo lo ha llegado a saber y por qué, mejorando su comprensión acerca de los procesos que llevan a los científicos a generar conocimiento (Programa $\mathrm{ECBI}^{4}$ ). Aumenta así la comprensión científica del alumno, su participación y motivación en actividades científicas, mejorándose el nivel de la educación general (IAP 2006; Schwartz \& Crawford 2006). Por otra parte, los alumnos necesitan aprender a organizar y regular su propio aprendizaje, ya sea individual o en grupos. La actual visión acerca de cómo ocurre el aprendizaje en ciencias -según la cual el aprendizaje es entendido como una construcción y reconstrucción del conocimiento por

$4 \quad$ Programa de Educación en Ciencias basada en la Indagación. 
parte de los alumnos- coincide con la indagación científica como enfoque pedagógico para la enseñanza de las ciencias (IAP 2006).

El uso de la indagación como enfoque pedagógico no sólo debiera llevar a una mayor comprensión de conceptos científicos y al desarrollo de habilidades científicas, sino también a una mayor comprensión acerca de la Naturaleza de la Ciencia, es decir, a la comprensión del desarrollo del conocimiento científico y su relación con la sociedad. Esto último, declarado como parte del concepto de competencia científica, es de especial relevancia si se pretende que los futuros ciudadanos tomen decisiones de manera informada en ámbitos sociales o personales relacionados con la ciencia (Abd-el-Khalick et al. 2004).

En resumen, sin la comprensión de las cualidades y supuestos del conocimiento científico (Naturaleza de las Ciencias) y del proceso a partir del cual este conocimiento es generado (Indagación Científica) el alumno está limitado en la construcción de su imagen de ciencias, la cual posiblemente se remitirá a una serie de datos aislados, sin un contexto que los haga relevantes, aplicables y significativos (Schwartz \& Crawford 2006). La relación de este enfoque pedagógico y el desarrollo de competencia científica es evidente (Abell et al. 2006; NRC 2002) (ver figura 1).

\section{Figura 1}

Relación entre Indagación Científica y el desarrollo de Competencia Científica

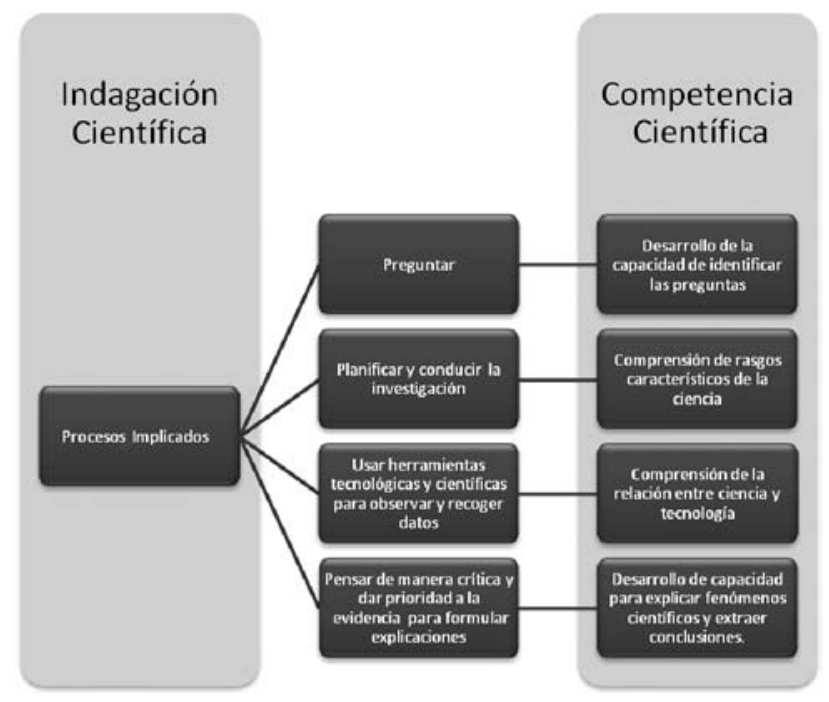

En relación a los tipos de indagación científica, es posible visualizar un gradiente continuo de acuerdo al grado de independencia que tiene el alumno en el planteamiento de preguntas y respuestas (Windschitl 2003; Herron 1971, en Zion et al. 2004). Así, se avanza desde las experiencias de confirmación, hasta la indagación abierta o independiente, identificándose niveles intermedios como indagación estructurada y guiada. En 
el nivel más alto -indagación abierta- los profesores permiten plantear al alumno sus propias preguntas y diseñar su propia investigación, significando una mayor oportunidad de desarrollar habilidades y actitudes científicas en el alumno (NRC 2002). Un alumno que tenga como desafío el diseño de un experimento tendrá evidentemente más posibilidades de desarrollar capacidades científicas que aquel que sólo se limita a seguir las instrucciones para la ejecución de un experimento ya dado. Así, en la educación secundaria, una aproximación a la indagación abierta pareciera ser la más provechosa para el desarrollo cognitivo del alumno.

El uso de la indagación científica en la enseñanza de las ciencias es promovido hoy en día por la comunidad científica internacional: desde el año 2000, las academias de ciencias del Mundo, representadas por el Inter Academy Panel on International Issues (IAP), han llamado a los científicos a generar Programas de Educación en Ciencias Basada en la Indagación en la enseñanza primaria como una manera de mejorar la calidad y el significado en la educación científica (Programa ECBI; IAP 2005).

El rol del docente en la indagación científica en contextos vulnerables. La indagación científica como modelo de enseñanza parece ser especialmente beneficiosa en contextos escolares vulnerables. Así lo describen dos estudios de caso independientes, en latitudes diferentes, que describen las prácticas de profesoras que trabajan en liceos pobres suburbanos en Pakistán y EE.UU. (ver Haberman 2006 y Zahur et al. 2002, respectivamente). En ambos estudios las docentes presentan una serie de características comunes: en lo pedagógico, ellas construyen la clase a partir de los intereses y preguntas de los propios alumnos invitándolos a generar preguntas e hipótesis acerca de cuestiones cotidianas y de su entorno, para posteriormente apoyarlos en la elaboración y ejecución de diseños experimentales orientados a probar sus hipótesis y dar respuesta a sus interrogantes, a través del trabajo en grupo, la argumentación, el diálogo y el debate, así como a través de la exploración directa y experimentación con materiales. En cuanto a sus creencias, ambas docentes presentan resultados similares entre sí: en ambos casos, las profesoras le asignan una gran importancia al logro de aprendizajes reales en los alumnos, que puedan aplicar a su vida cotidiana. Además, confían en sus alumnos y presentan altas expectativas de ellos. Uno de los estudios describe con detalle el rol que la profesora le asigna a la educación en ciencias: ella ve a los alumnos como factores de cambio social, no sólo después de egresados, sino durante el tiempo que son alumnos secundarios, por ejemplo, a través de proyectos concretos, como la explotación responsable, recuperación y cuidado de recursos naturales en el barrio en que viven. Se describen además logros en el ámbito del desarrollo personal del alumno, como autoestima y autonomía, así como un gran impacto en la comunidad. Estos rasgos coinciden en parte con las prácticas pedagógicas características de las escuelas efectivas chilenas, las cuales son descritas como motivadoras y cercanas a la vida cotidiana de los alumnos, con propósitos claros, exigentes, con predominio de refuerzos positivos, y que dan prioridad a la expresión de ideas, razonamiento lógico y autonomía de los niños, entre otros (UNICEF 2004; Raczynski \& Muñoz 2007).

Implementación de la metodología indagatoria en la educación en ciencias en Chile. En Chile, el Programa ECBI, del Ministerio de Educación, la Universidad de Chile y la Academia Chilena de Ciencias es un ejemplo concreto de la implementación de esta 
metodología en la enseñanza básica. Las evaluaciones realizadas al día de hoy -eminentemente cualitativas- indican buenos resultados, entre los que destacan, en los alumnos: "cambios positivos en el clima del aula con mejores relaciones colaborativas y de trabajo equipo, progreso en la autonomía del aprendizaje de los alumnos, aumento en la motivación por aprender y saber más y una buena participación de alumnos con dificultades de aprendizaje (Programa ECBI, 2007)", así como avance sustancial en su conocimiento científico (contenido y habilidades), además de una mejora significativa en la capacidad de expresarse en forma oral y escrita.

Al igual que ECBI, el Proyecto MECIBA, "Modelo de desarrollo profesional docente entre pares, para fortalecer la calidad de la enseñanza de las Ciencias Naturales en Kinder y Enseñanza Básica", de la P. Universidad Católica de Valparaíso, ha contribuido a la implementación de este tipo de enseñanza a nivel de educación básica. Como consecuencia de la aplicación de este proyecto, se ha observado una mejora en el aprendizaje de los procesos científicos en los escolares-alumnos de los profesores participantes del Programa (MECIBA 2006), lo que se ha traducido, por ejemplo, en los resultados del SIMCE de $4^{\circ}$ básico del año 2007: las escuelas que participaron en MECIBA con docentes de kinder a $4^{\circ}$ básico subieron en promedio 17.60 puntos, en circunstancias que las otras escuelas de la corporación bajaron 4.90. De las 10 escuelas MECIBA, seis subieron al menos 20 puntos, con dos subiendo más de 40 puntos.

Sin duda, tanto el Programa ECBI como MECIBA están colaborando de manera importante, aunque con una cobertura limitada, al mejoramiento de la enseñanza de las ciencias en nuestro país. Cabe destacar que ambas iniciativas se desarrollan en escuelas municipalizadas y, en buena parte, en contextos de alta vulnerabilidad. Su implementación no sólo ha impactado en el aprendizaje, sino por sobre todo en la autoestima de los alumnos, su motivación por aprender y la expectativa de profesores y padres. Este cambio de visión de los niños, sus familias y su entorno es claramente un impulso para la movilidad social.

No obstante, los alumnos ECBI y de profesores MECIBA llegarán a la enseñanza media y se verán enfrentados mayoritariamente a una enseñanza frontal/tradicional de las ciencias, en donde no podrán seguir desarrollando las competencias adquiridas en los programas mencionados. Más aún, en Chile no existe ningún programa que promueva la enseñanza de las ciencias en enseñanza media a través de actividades de indagación científica. Surge entonces la pregunta: ¿Es posible implementar esta metodología en la educación secundaria?

Características del profesorado que pueden influir en la implementación exitosa de la metodología indagatoria en las aulas a nivel de educación secundaria. En general, y a pesar de estar explícita incluso en los currículos oficiales de muchos países, la enseñanza de las ciencias basada en la indagación científica parece estar ausente en la mayoría de las aulas de ciencia, y en aquellas en que existe la indagación del tipo confirmatoria y estructurada suele ser la más común (Windschitl 2003). Los factores que, según la literatura, pueden influir en que la implementación de esta metodología en las aulas tenga éxito en el logro de aprendizajes científicos significativos, particularmente a nivel de educación secundaria, son los siguientes: (a) Competencia científica del profesor: Como bien se indicó anteriormente, el concepto de competencia científica implica que un individuo, además de demostrar conocimiento, habilidades y actitudes científicas, comprende los 
rasgos característicos de la ciencia -esto es, la naturaleza de la ciencia- entendida como una forma del conocimiento y la investigación humanos. Según Windschitl (2003) aquellos profesores que carecen de este tipo de conocimiento son menos capaces de incluir este aspecto en su enseñanza, a su vez que se encuentran limitados en el aprendizaje de nuevos conocimientos en el área. Por otra parte, existen evidencias que muestran que profesores que tienen poco dominio de la disciplina -o de aspectos particulares de la misma- suelen realizar sus clases en formatos más tradicionales, remitiéndose a reproducir el contenido de un texto o documento y negando a los alumnos cualquier posibilidad de indagación, frente al temor de hacer evidente su ignorancia (Vergara 2006). (b) Experiencia en investigación e indagación científica: Un elemento crítico para la comprensión y aplicación de la indagación científica de nivel superior por parte del profesor -a pesar de que aún no hay evidencia suficiente para afirmarlo- parece ser la experiencia que este haya tenido previamente en investigación científica (Abd-el-Khalick et al. 2004; Schwartz y Crawford 2006). Según un estudio de Windschitl (2003), los profesores que mejor y más implementaban metodologías de indagación científica en su sala de clases eran aquellos que habían tenido experiencias significativas de investigación científica durante sus estudios de pregrado o durante su vida profesional.

Un estudio exploratorio realizado en nuestro país muestra similares resultados. En él, Castro et al. (2005) investigaron la relación existente entre la experiencia en investigación del profesor y los niveles de alfabetización científica alcanzados por sus estudiantes de $4^{\circ}$ año medio. En la investigación participaron 30 profesores de biología de la V Región, seleccionados a través de un cuestionario descriptivo -referente a la formación académica del profesor y de su experiencia en investigación- y sus respectivos alumnos de $4^{\circ}$ año medio $(n=534)$, quienes fueron sometidos a una Prueba tipo PISA. De los 30 profesores, 10 pertenecían a colegios particulares subvencionados, y tenían experiencia en investigación, 10 pertenecían al mismo tipo de establecimiento, pero sin este tipo de experiencia, y los 10 restantes eran de colegios particulares pagados, y no tenían experiencia en investigación. Al comparar los resultados de los alumnos de colegios particulares subvencionados entre sí, los autores pudieron afirmar con un $95 \%$ de certeza que los alumnos de profesores que tienen experiencia en investigación científica obtienen mayores resultados en la prueba tipo PISA, lo que supone que dichos profesores desarrollan más habilidades científicas en sus alumnos. A su vez, y aunque la diferencia de resultados encontrada entre los alumnos de colegios particulares subvencionados de profesores con experiencia en investigación versus aquellos alumnos de colegios particulares pagados (con profesores sin experiencia en investigación) es menor, los alumnos de profesores con experiencia parecieran mostrar mejores resultados y menos dispersión. Esto último entrega pistas respecto a que, en presencia de profesores efectivos, la condición socioeconómica no es del todo determinante en la adquisición de aprendizajes por los estudiantes. Sin embargo, se requiere de futuras investigaciones que profundicen y descubran a cabalidad cómo se relacionan las variables implicadas. (c) Concepciones de ciencia, enseñanza de las ciencias e indagación científica: Abd-el-Khalick et al. (2004) y Windschitl (2003) describen las dificultades que diversos países han tenido en la aplicación de la metodología indagatoria en sus escuelas. Respecto de las concepciones científicas y epistemológicas de los profesores varios estudios han demostrado una tendencia mayoritaria a tener una visión absolutista/positivista del conocimiento científico (Suárez y López-Guazo 1993; Porlán \& Martín 2002; Porlán et al. 1998; Sanmartí 2002; Schwartz \& Crawford 2006). 
Tal situación supone un obstáculo para un proceso de enseñanza de las ciencias desde una visión constructivista. En palabras de Pope y Gilbert (1983, citado en Porlán et al. 1998: 274): "La concepción positivista y empírico-inductivista de las ciencias está en consonancia con la versión absolutista de la verdad y del conocimiento. Así, los profesores que sostienen esta concepción de ciencia, del contenido del currículo y de la manera de enseñar a los estudiantes pondrán poco o ningún énfasis en sus concepciones y en su participación activa". Por su parte, y en el contexto indagatorio, a menudo el profesor se conforma sólo con el aspecto procedimental del "hacer ciencias", restándole importancia al hecho de que los alumnos efectivamente reflexionen y comprendan lo que están haciendo. En palabras de Schwartz \& Crawford (2006), la indagación científica sólo promueve una adecuada visión de ciencia, si se le acompaña por un proceso de reflexión explícita, acerca de las actividades realizadas. Un segundo obstáculo es la creencia en muchos profesores de que sólo es posible emplear metodologías indagatorias con alumnos sobresalientes, y de que, en general, es una metodología difícil de manejar en el aula (Abd-el-Khalick et al. 2004). En ese sentido, un concepto inadecuado de indagación científica como herramienta pedagógica también parece influir.

\section{EL ROL DE LAS UNIVERSIDADES FORMADORAS DE PROFESORES EN EL MEJORAMIENTO DE LA CALIDAD Y LA EQUIDAD DE LA EDUCACION CIENTIFICA A NIVEL SECUNDARIO}

A diferencia de iniciativas anteriores, la Nueva Reforma Educacional Chilena pone énfasis en la calidad y equidad de la educación más que en la cobertura, especialmente en los niveles de enseñaza básica y media (MINEDUC 2007; Contreras y Elaqua 2005). En este contexto, surge la necesidad de mejorar la formación docente, a la vez que se reconoce que éste ha sido uno de los aspectos que la Reforma Educacional de 1990 no ha podido mejorar de manera sustancial (Aylwin 2000; Raczynski y Muñoz 2007). Un estudio de fines de los 90 muestra que más del $80 \%$ de nuestros profesores de enseñanza media realiza clases expositivas, privilegiando la transferencia verbal y la memorización pasiva como metodología de enseñanza-aprendizaje. De este modo se estimula la capacidad para recordar respuestas hechas por sobre la capacidad de elaborar, a través de una reflexión personal, la respuesta a una pregunta (Chacón 2003; Schiefelbein y Schiefelbein 2000). Bajo este modelo, además, disminuyen al mínimo las posibilidades de los alumnos de discutir y tomar conciencia de sus conocimientos previos, de reflexionar sobre ideas alternativas a las del profesor o del texto, aplicar el contenido aprendido a la vida cotidiana y expresar su opinión, mejorando la comunicación oral y escrita, todo lo cual se relaciona estrechamente con una mala calidad de los aprendizajes (Schiefelbein y Schiefelbein 2000). Por su parte, la OECD, Organización Económica de Cooperación y Desarrollo, en un informe reciente sobre el estado de la Educación en el Mundo, enfatiza la necesidad de dar prioridad a la calidad de los profesores antes que a su cantidad, argumentando que "sólidas investigaciones evidencian que la calidad del profesorado y su docencia influyen de forma determinante en los resultados de los estudiantes" (OECD, 2004: 6; Albertini et al. 2005). La OECD, al igual que nuestro país, apuesta a la calidad de la educación más que a la cobertura. 
Asumiendo que la indagación científica puede ser un camino hacia el mejoramiento de la calidad y la equidad de los aprendizajes científicos en la educación secundaria, y retomando los ya mencionados factores que pueden influir en que esta metodología se implemente en este nivel de escolaridad, surgen algunas preguntas. Más que responderlas -para lo cual harían falta cuidadosos estudios- nuestra intención es someterlas a discusión, de modo de promover la reflexión y, por qué no, nuevas líneas de investigación, que nos den luces acerca de cómo guiar la formación docente para el mejoramiento de la educación científica en nuestro país.

De la competencia científica del profesor. ¿En qué medida la formación inicial de docentes del área científica promueve el desarrollo de competencia científica en sus egresados? Si bien no contamos con suficientes antecedentes aún para responder, podemos entregar algunos datos que nos permiten hacer una breve aproximación. El laboratorio de ciencias universitario es uno de los ejes centrales de la formación disciplinaria en la formación del profesor. González-Weil et al. (2008) ${ }^{5}$ investigaron de manera exploratoria, a partir de la observación de más de 20 horas de clase, las acciones del profesor universitario que podrían promover competencias científicas en el contexto del trabajo de laboratorio. Los resultados preliminares de este estudio muestran que, en promedio, el tiempo dedicado a este tipo de acciones no sobrepasa el $15 \%$ de la clase. De este porcentaje, las conductas más habituales se relacionan con la enseñanza de procedimientos científicos y técnicas, la recolección y representación de datos y un gran énfasis en la rigurosidad científica. Del mismo modo, los procesos científicos mayormente realizados por los alumnos se remiten a la observación, la clasificación y la comunicación. Procesos científicos de orden intermedio o superior, como inferir, formular hipótesis, predecir, diseñar experimentos o formular modelos, son prácticamente inexistentes. Así, nuestros resultados dan cuenta de un modelo mayoritariamente tradicional de docencia experimental, en donde el laboratorio, aun a nivel universitario, se transforma en una instancia meramente procedimental, en la cual los alumnos se limitan a "seguir una receta", sin mayores espacios para la reflexión y la indagación científica.

En el mismo contexto, y al evaluar los niveles cognitivos que demandan en los alumnos las preguntas del profesor, los datos muestran que de las 418 preguntas registradas, más del $70 \%$ corresponden a preguntas que promueven los niveles cognitivos más básicos: memorizar (52\%) y comprender (26\%), siendo el tipo de conocimiento más frecuentemente promovido también el tipo más simple: conocimiento factual. Tipos de procesos cognitivos más elevados como evaluar y crear prácticamente no son promovidos, lo mismo que el tipo de conocimiento metacognitivo. Lo anterior demuestra que, en términos de la calidad de las preguntas realizadas por el profesor, los resultados coinciden con la descripción que se hace de los laboratorios en la literatura, en donde se reporta que muy a menudo los docentes desarrollan actividades de laboratorio que para los alumnos son rutinarias, en las cuales la interacción profesor-alumno se limita

5 González-Weil, C., Cáceres, P., Cuevas, K., Martínez, M., Martínez C., Muñoz, L., Álvarez, S., Castillo, P. \& Ramírez, J. (2008). ¿Es el laboratorio un espacio para el desarrollo de competencia científica?: el caso de los alumnos de Pedagogía en Ciencias. En: Informe de Investigación del Proyecto DI 122.799 /2007 (trabajo aún no publicado). 
a preguntas y respuestas de bajo nivel cognitivo, y en su mayoría meramente procedimentales (González-Weil et al. 2008) ${ }^{6}$.

De la experiencia en investigación e indagación científica. ¿En qué medida la formación inicial de docentes del área científica otorga oportunidades a sus estudiantes de involucrarse en proyectos de indagación abierta o investigación, de modo de adquirir este tipo de experiencia? Si consideramos que la mejor manera de que los individuos aprendan ciencia es haciendo ciencia, esto es, experimentar la ciencia como un acto de investigación (Hodson 1994) y que, por otra parte, existe una tendencia a enseñar de la manera en que se ha sido enseñado (Albertini et al. 2005), parece obvio que los profesores de ciencia en formación han de tener un acercamiento real a la actividad científica. En este sentido, se debe dar al futuro docente la oportunidad de poder producir conocimiento científico, más que reproducirlo (Hodson 1994). Esta idea no es nueva. Ya en 1990 Krugly-Smolska planteaba al respecto que es altamente recomendable que los estudiantes de pedagogía en ciencias tuvieran la oportunidad de conducir o de ser parte de un proyecto completo de investigación científica (Krugly-Smolska 1990: 479). En el mismo sentido, un estudio realizado en Taiwán con estudiantes de primer año de pedagogía en ciencias reveló que éstos tenían una actitud más positiva hacia las ciencias, así como un mayor grado de alfabetización científica si habían tenido, de manera previa, experiencia en actividades científicas (Chin 2005). En cuanto a la realidad de nuestro país, Albertini et al. (2005) indican como una de las causas de las falencias de nuestra educación científica la obsolescencia y baja especialización en las disciplinas científicas que se enseñan en algunas instituciones formadoras de profesores, debido principalmente a la falta de actividad científica en ellas. Consecuentemente, y según estos autores, las universidades con alta producción científica tendrían mayores posibilidades de entregar una mejor formación científica a sus estudiantes, en relación a la formación netamente pedagógica (Albertini et al. 2005). No obstante lo anterior, parece ser que en muchos casos, para los estudiantes de pedagogía en ciencias las oportunidades de participar como ayudantes en proyectos de investigación, o más aun, de guiar su propia investigación científica, son mínimas. A primera vista, da la sensación de que la formación de estos profesionales se ubica lejos de los laboratorios de investigación $\mathrm{y}$, a la hora de elegir ayudantes, los científicos a menudo prefieren a alumnos de licenciatura en ciencias.

De las concepciones de indagación científica, ciencia y enseñanza de las ciencias. ¿Cuáles son las concepciones de ciencia que promueven las universidades en sus estudiantes de pedagogía en ciencias? Respecto de esta pregunta, hay evidencias internacionales que indican que, en general, la visión de ciencia entregada a nivel de formación inicial corresponde a la visión común de ciencia. En su estudio sobre el conocimiento profesional y la epistemología de los profesores, Porlán et al. (1998) relatan los resultados de cuatro estudios empíricos realizados en torno a esta temática. Respecto a la imagen de ciencia, Porlán y sus colegas concluyen que, en general, predomina la creencia de que

6 González-Weil, C., Cáceres, P., Quiñones, P., Carriel, O., Tapia, N. \& Villegas, V. (2008). Preguntas del profesor y procesos de retroalimentación a las respuestas de los alumnos en las clases de laboratorio de ciencias universitario: el caso de los estudiantes de pedagogía en ciencias. En: Informe de Investigación del Proyecto DI 122.799 /2007 (trabajo aún no publicado). 
la observación y la experimentación permiten obtener -por inducción- un fiel reflejo de la realidad, obviando la subjetividad, historicidad y contextualización de los procesos de generación de conocimiento científico. Dichos resultados coinciden con los de Abd-elKhalick (2006), quien en un estudio con 153 estudiantes universitarios (mayoritariamente de carreras científicas) concluye que en la mayoría de los casos persiste una imagen de ciencia ingenua e inadecuada, entendiéndose por conocimiento científico un derivado directo de lo empírico, que está generado a partir de un procedimiento rígido tipo "receta", llamada método científico. Una visión similar es la que describe Windschitl (2003) respecto de la idea de que estudiantes de pedagogía en ciencias sin experiencia previa en investigación tienen sobre indagación científica. Las causas de lo anterior están probablemente en las pocas posibilidades que, en general, se ofrecen a los estudiantes de pedagogía en ciencias de participar en procesos de investigación e indagación científica, en la metodología tradicional de enseñanza a la que se ven sometidos en las aulas universitarias y a la ausencia explícita de la naturaleza de la ciencia como contenido curricular. Lo anterior no hace más que reforzar la permanencia del modelo tradicional de enseñanza, a la vez que obstaculiza la implementación de metodologías innovadoras, como la enseñanza de las ciencias basada en la indagación.

Finalmente, ¿es posible pensar la educación científica como apoyo a la movilidad social? La educación secundaria parece ser un período determinante en el destino de las personas. Durante este periodo, y a través de la educación científica, es posible desarrollar en los alumnos no sólo una comprensión del mundo en que vivimos, sino la capacidad de ser crítico y riguroso, de poder formarse su propia opinión, de tomar decisiones basadas en evidencia, de trabajar en equipo y de contribuir a mejorar la calidad de vida, tanto propia como del entorno, a través de la habilidad para resolver los diversos problemas a los que se enfrenten, como el cuidado del medio ambiente y de la salud. Para ello se necesita contar con oportunidades para desplegar y poner en práctica los propios recursos y habilidades superiores, para enfrentar la complejidad de la construcción del conocimiento científico y experimentar lo que ello significa en su ser persona, al tomar decisiones responsables y vivir las implicancias de ello.

La evidencia muestra, sin embargo, que en nuestro país la calidad de los aprendizajes en ciencia es insuficiente y poco equitativa, predominando una formación memorística, reproductiva, instructiva, depositaria y aislada de la vida y los problemas cotidianos. Frente a esta realidad, el rol del profesor es crucial en el cambio hacia una enseñanza de calidad; sin embargo, la responsabilidad de transformar las prácticas pedagógicas no es exclusiva de los docentes, sino es una problemática país, que debe ser abordada por las diferentes instancias educativas, principalmente las IES que tienen por labor la formación inicial del profesorado y la generación de conocimiento. En esta instancia es donde ha de cultivarse el desafío de generar estrategias pertinentes e innovadoras para poder contribuir a una transformación en la calidad de los aprendizajes y de la formación de formadores. Esta tarea demanda una vinculación y mirada colaborativa, que sea capaz de integrar y asumir la complejidad de la formación del profesor de ciencias, cuyos aprendizajes integran necesariamente el conocimiento científico y pedagógico (Labarrere 2003). A partir de las reflexiones anteriores, queda claro que, si queremos mejorar la calidad, y por sobre todo la equidad de la educación en ciencias, la formación de profesores de ciencia no debiera estar sólo enfocada a la comprensión de contenidos científicos y pedagógicos. 
Para ello, los planes de estudio deben ofrecer, en concreto, a los estudiantes elementos que los capaciten para aprender, construir y manejar el conocimiento, con el fin de que comprendan conceptos y teorías propias de su especialidad (Hernández 1996). De este modo, es probable que mientras en la formación inicial de profesores de ciencia se siga poniendo el enfoque en los contenidos más que en el quehacer científico, mientras no se invite a los futuros profesores a ser parte de proyectos de investigación y mientras no se incluya de manera explícita la naturaleza de la ciencia y la indagación científica como objeto de reflexión, las concepciones epistemológicas del profesorado seguirán adscritas a un modelo tradicional, que entiende la ciencia como un conjunto cerrado de conocimientos, lo que repercute en una enseñanza también tradicional, basada en contenidos más que en el desarrollo competencias. Actualmente, tanto a nivel de propuestas de universidades y del MINEDUC, como a través de la Academia Chilena de Ciencias, se están desarrollando iniciativas de innovación en la enseñanza de las ciencias originadas internacionalmente y extrapoladas a la realidad sociocultural, política y económica del país, las cuales están teniendo importantes logros en la transformación de prácticas pedagógicas y en cuanto a calidad de aprendizajes (Albertini et al. 2005). No obstante estas experiencias, y como se comentaba en un comienzo, existen en Chile docentes secundarios que por propia iniciativa se atreven a traspasar las fronteras tradicionales de la enseñanza de las ciencias y que nos pueden dar luces acerca de las características del rol docente. En este sentido, resultaría interesante explorar ¿qué caracteriza estas formas innovadoras de enseñanza de las ciencias?, ¿cuáles son los orígenes de estas experiencias?, ¿qué concepciones son la base de estas iniciativas?, y ante nuevos hallazgos, ¿cómo podría transformarse la formación del profesor de ciencias para contribuir cualitativa y equitativamente al desarrollo de todos y todas? La invitación a responder, queda hecha.

\section{BIBLIOGRAFIA}

Abd-el-Khalick, F. (2006). Over and over again: college students' views of nature of science. En: Flick, L \& Lederman, N. (eds.), Scientific inquiry and the nature of science: Implications for teaching, learning, and teacher education, pp. 389-425. Netherlands: Springer.

Abd-el-Khalick, F., S. Boujaoude, R. Duschl, R. Mamlok-Naaman, A. Hofstein, M. Niaz, D. Treagust \& Tuan Hsiao-Lin (2004). Inquiry in Science Education: International Perspectives. Science Education 88: 397-419.

Abell, S., D. Smith \& M. Volkmann (2006). Inquiry in Science Teacher Education. En: Flick, L y N. Lederman (eds.). Scientific inquiry and the nature of science: Implications for teaching, learning, and teacher education (pp. 389-425). Netherlands: Springer.

Albertini, R., G. Cárdenas-Jirón, J. Babel, G. Díaz Véliz, J. Eyzaguirre, A. Labra y R. Lewin (2005). Enseñanza de las ciencias a nivel escolar y formación en ciencia en el pregrado universitario. En: Ureta, T., J. Babul, S. Martínez y J. Allende. Análisis y Proyecciones de la Ciencia Chilena 2005. Academia de Ciencias. Recuperado el 03-04-08 de: http://www.academia-ciencias. $\mathrm{cl} /$ ?module=investig.

Aylwin, M. (2000). Ética, Desarrollo Humano y Economía: El Desafío de la Inequidad en Educación. Seminario BID, Dic. 2000. Recuperado el 20-03-07 de: http://www.iadb.org/etica/ documentos/dc_ayl_etica.pdf.

Castro, M., R. Cerón, C. Eberlein y J. Reyes (2005). Experiencia en Investigación del Profesor de Biología y su relación con la Alfabetización Científica de sus alumnos de $4^{\circ} \mathrm{EM}$, según Prueba tipo PISA aplicada en 30 Establecimientos Educacionales de la V Región. Trabajo de 
titulación para optar al grado de Licenciado en Educación y al título de Profesor de Biología y Cs. Naturales. Pontificia Universidad Católica de Valparaíso.

Chacón (2003). Calidad y Equidad en la Educación Media. Santiago de Chile: Depesex/Bcn/Serie Informes, Año XIV, $\mathrm{N}^{\circ}$ 129. Recuperado el 07-08-07 de: http://www.bcn.cl/bibliodigital/pben/ informes/estudios_pdf_informes/nro129.pdf

Chin, C. (2005). First-year pre-service teachers in Taiwan - Do they enter the teacher program with satisfactory scientific literacy and attitudes toward science? Int. J. Sci. Educ. 27 (13): 1549-1570.

Contreras, D. y G. Elaqua (2005). Calidad y equidad: el desafío de la educación chilena. Diario Financiero 9 de mayo de 2005.

Hernández, M. (1996). La historia de la ciencia y la formación de científicos. Revista Perfiles Educativos 18 (73): 33-39.

Gil, D. y A. Vilches (2001). Una alfabetización científica para el siglo XXI. Investigación en la Escuela 43: 27-37.

Haberman, M. (2006). The special role of science teaching in schools serving diverse children in urban poverty. En: Flick, L \& N. Lederman (eds.), Scientific inquiry and the nature of science: Implications for teaching, learning, and teacher education (pp. 37-53) Netherlands: Springer.

Harlen, W. (2002). Evaluar la alfabetización científica en el programa de la OECD para la evaluación internacional de estudiantes (PISA). Enseñanza de las Ciencias 20 (2): 209-216.

Hattie, J. (2003). Teachers make a difference: What is the research evidence? Paper presented at the Australian Council for Educational Research Annual Conference on Building Teacher Quality, Melbourne.

Hodson, D. (1994). Hacia un enfoque más crítico del trabajo de laboratorio. Enseñanza de las Ciencias 12 (3): 299-313.

IAP (2005). Science Education: Workshop Evaluation of Inquiry-Based Science Education Programme (Stockholm, 21-23 September 2005). Recuperado el 12-04-06 de: http://www.interacademies. net/Object.File/Master/5/180/Conclusions_Stockholm05.pdf

IAP (2006). Report of the Working Group on International Collaboration in the Evaluation of Inquiry-Based Science Education (IBSE) programs. Recuperado el 12-04-06 de: http://www. interacademies.net/Object.File/Master/7/078/IBSE\%20Rep.pdf.

Krugly-Smolska, E. (1990). Scientific Literacy in developed and developing countries. Int. J. Sci. Educ. 12 (5): 473-480.

Labarrere, A. (2003). La formación de profesores de ciencia: un enfoque desde la teoría de la profesionalización temprana y de los procesos de orientación. Revista Extramuros 2 (2): 21-32.

Macedo, B. y R. Katzkowicz (2002). Repensando la Educación Secundaria. En UNESCO/OREALC. Educación secundaria. Un camino para el desarrollo humano. Santiago: OREALC.

Marcelo, C. (2006). La formación docente en la sociedad del conocimiento y la información: avances y temas pendientes. Ponencia presentada al IV Encuentro Internacional de KIPUS. Políticas públicas y formación docente, Isla Margarita (Venezuela, 4-6 de octubre, 2006).

MECIBA (2006). Programa de Desarrollo Profesional Docente en Ciencias. Recuperado el 14-1106 de: http://www.meciba.cl/index_meciba.htm.

MINEDUC (2003). Resultados Estudio PISA. Habilidades para la Lectura en el Mundo de Mañana. Resumen Ejecutivo. Estudios Internacionales UCE-MINEDUC. Recuperado el 14-07-05 de: http://www.sectormatematica.cl/pisa/resumen_ejecutivo.pdf

MINEDUC (2006). PISA 2006: Rendimientos de estudiantes de 15 años en Ciencias, Lectura y Matemática. Unidad de Currículum y Evaluación.

MINEDUC. (2007). Mensaje de S.E. la Presidenta de la República con el que inicia un Proyecto de Ley que establece la Ley General de Educación. En: Proyecto de Ley General de Educa- 
ción. Recuperado el 04-05-08 de: http://www.mineduc.cl/biblio/documento/200704111034430. proyecto $\% 20$ de $\% 20$ ley.pdf.

NRC: National Research Council (2002). Inquiry and the National Science Education Standards: A Guide for Teaching and Learning. Washington, D.C.: National Academy Press.

OECD (2004). Teachers Matter: Attracting, Developing and Retaining Effective Teachers. Recuperado el 27-05-07 de www.oecd.org/bookshop

OECD (2006). Assessing Scientific, Reading and Mathematical Literacy: A framework for PISA 2006. Recuperado el 20-12-06 de http://www.oecd.org/dataoecd/63/35/37464175.pdf

Porlán, Aristía, Rivero y Martín del Pozo (1998). Conocimiento profesional y epistemológico de los profesores II: estudios empíricos y conclusiones. Enseñanza de las Ciencias 16 (2): 271-288

Porlán \& Martín (2002). Spanish Teachers' epistemological and Scientific Conceptions: implications for teacher education. European Journal of Teacher Education 25 (2): 151-169.

Programa ECBI (2007). En Internet bajo: www.ecbichile.cl. Recuperado el 20-03-07.

Raczynski, D. y G. Muñoz (2007). Reforma Educacional Chilena: El difícil equilibrio entre la macro y la micropolítica. Serie Estudios Socio / Económicos $\mathrm{N}^{\circ} 31$.

Román, M. (2003). Por qué los docentes no pueden desarrollar procesos de enseñanza de calidad en contextos sociales vulnerables. Persona y Sociedad 17 (1): 113-120.

Sanmartí, N. (2002). Didáctica de las Ciencias en la Educación Secundaria Obligatoria. Madrid: Editorial Síntesis.

Schiefelbein, E. y P. Schiefelbein (2000). Determinantes de la calidad: ¿qué falta mejorar? Revista Perspectivas 4 (1): 37-64.

Schwartz, R. \& B. Crawford (2006). Authentic Scientific Inquiry as Context for Teaching Nature of Science. En: Flick, L \& N. Lederman (eds.), Scientific inquiry and the nature of science: Implications for teaching, learning, and teacher education, pp. 389-425. Netherlands: Springer.

SIMCE (2008). Resultados Nacionales SIMCE 2007. SIMCE. Unidad de Currículum y Evaluación (UCE), Ministerio de Educación, Santiago, Chile. Recuperado el 15-06-08 de http://www. simce.cl/index.php?id=247.

Suárez L. y L. López-Guazo (1993). Metodología de la Enseñanza de las Ciencias. Perfiles Educativos 62 .

Tedesco, J. y N. López (2002). Desafíos a la educación secundaria en América Latina. Revista de la CEPAL 76: 55-69.

UNESCO-ICSU (1999). Declaración de Budapest. Conferencia Mundial sobre la Ciencia para el Siglo XXI: Un nuevo compromiso. Recuperado el 12-01-07 de http://www.oei.es/salactsi/ budapestdec.htm

UNICEF (2004). ¿Quién dijo que no se puede? Escuelas efectivas en sectores de pobreza. Santiago: UNICEF.

Vergara, C. (2006). Concepciones sobre la enseñanza y el aprendizaje en profesores de biología: Coherencia entre el discurso y la práctica de aula. Tesis doctoral para optar al grado de Doctor en Ciencias de la Educación de la Pontificia Universidad Católica de Chile.

Windschitl, M. (2003). Inquiry Projects in Science Teacher Education: What Can Investigative Experiences Reveal About Teacher Thinking and Eventual Classroom Practice? Science Education 87: 112-143.

Zahur, R., A. C. Barton \& B. R. Upadhyay (2002). Science education for empowerment and social change: a case study of a teacher educator in urban Pakistan. Int. J. Sci. Educ. 24 (9), 899-917.

Zion, M., M. Slezak, D. Shapira, E. Link, N. Bashan, M. Brumer, T. Orian, R. Nussinowitz, D. Court, B. Agrest, R. Mendelovici \& N. Valanides (2004). Dynamic, open inquiry in biology learning. Science Education 88 (5): 728-753. 